\title{
Cenário da prevalência e condições socioambientais associadas às geo-helmintíases no Brasil: Uma revisão integrativa da literatura
}

\author{
Scenario of prevalence and socio-environmental conditions associated with soil-transmitted \\ helminth infections in Brazil: An integrative review study \\ Escenario de prevalencia y condiciones socioambientales asociadas a la geohelmintiasis en Brasil: \\ Revisión integradora de la literatura
}

Recebido: 13/04/2021 | Revisado: 19/04/2021 | Aceito: 20/04/2021 | Publicado: 05/05/2021

Marilene Aparecida Moreira
ORCID: https://orcid.org/0000-0002-1943-9000
Universidade do Estado de Mato Grosso, Brasil
E-mail: jmhemala@ @otmail.com
Andernice dos Santos Zanetti
ORCID: https://orcid.org/0000-0001-7888-8183
Universidade do Estado de Mato Grosso, Brasil
E-mail: andernice.zanetti@unemat.br
Lucas França de Barros
ORCID: https://orcid.org/0000-0001-5203-178X
Universidade do Estado de Mato Grosso, Brasil
E-mail: lucasmariano.f@ @mail.com
Luandra Abny Moreira da Cruz
ORCID: https://orcid.org/0000-0003-2954-8734
E-mail: jmhemala @hotmail.com
Antonio Francisco Malheiros
Instituto Federal de Educação, Ciência e Tecnologia de Mato Groso, Brasil
ORCID: https://orcid.org/0000-0001-8169-0557
Universidade do Estado de Mato Grosso, Brasil
E-mail: malheiros@unemat.br

\section{Resumo}

O objetivo foi conhecer a prevalência e condições socioambientais da população brasileira acometida por geohelmintos nos últimos dez anos. Estudo exploratório e descritivo de revisão integrativa de literatura com recorte temporal de 2010-2020. Busca realizada em quatro bancos de dados de acesso aberto. Estatística descritiva utilizada para os cálculos de prevalências dos geo-helmintos e dados socioambientais. Um total de 841.107 amostras humanas foram incluídas nos 55 estudos elegíveis para esta revisão. A prevalência combinada de geo-helmintos no país foi de 19\%. Ascaris lumbricoides (68\%) foi o mais prevalente, seguido por Ancilostomídeos (24\%) e Trichuris trichiura (8\%). Alta prevalência foi observada na região Centro-Oeste (22\%), Nordeste (19\%) e Norte (14\%). Saneamento precário, hábitos inadequados de higiene e baixa renda foram os fatores associados às geo-helmintíases mais reportados nos estudos. As infecções geo-helmínticas ainda são prevalentes no país embora apresente uma ligeira queda nos últimos cinco anos. Assim como a tendência mundial, Ascaris lumbricoides foi o geo-helminto mais prevalente. Dente os principais fatores associados às infecções, destacam-se o saneamento precário, hábitos inadequados de higiene e a baixa renda. Esperamos que os resultados desta revisão sirvam como uma contribuição para as políticas públicas e administrações regionais com programas de sensibilização da população acerca da prevenção e cuidados para não propagar a infecção, além de investimentos na infraestrutura no setor de coleta de lixo, rede de esgoto, tratamento de água e expandir a administração em massa de tratamentos antiparasitários no Brasil.

Palavras-chave: Helmintos; Ascaris lumbricoides; Trichuris trichiura; Ancilostomídeos.

\begin{abstract}
The objective was to know the prevalence and socio-environmental conditions of the Brazilian population affected by soil-transmitted helminth infections in the last ten years. Exploratory and descriptive study of integrative literature review with time frame from 2010-2020. Search performed in four open access databases. Descriptive statistics used for calculations of prevalence of soil-transmitted helminth and socio-environmental data. A total of 841,107 human samples were included in the 55 studies eligible for this review. The combined prevalence of soil-transmitted helminth in the country was $19 \%$. Ascaris lumbricoides (68\%) was the most prevalent, followed by hookworms (24\%) and Trichuris trichiura (8\%). High prevalence was observed in the Midwest (22\%), Northeast (19\%) and North (14\%). Poor sanitation, inadequate hygiene habits and low income were the factors associated with soil-transmitted helminth
\end{abstract}


infections most reported in the studies. Soil-transmitted helminth infections are still prevalent in the country although it has dropped slightly in the past five years. As with the global trend, Ascaris lumbricoides was the most prevalent soil-transmitted helminth. Among the main factors associated with infections, poor sanitation, inadequate hygiene habits and low income stand out. We hope that the results of this review will serve as a contribution to public policies and regional administrations with programs to raise awareness among the population about prevention and care not to spread the infection, in addition to investments in infrastructure in the garbage collection sector, sewage system, water treatment and expand the mass administration of antiparasitic treatments in Brazil.

Keywords: Helminths; Ascaris lumbricoides; Trichuris trichiura; Hookworms.

\section{Resumen}

El objetivo fue conocer la prevalencia y las condiciones socioambientales de la población brasileña afectada por geohelmintos en los últimos diez años. Estudio exploratorio y descriptivo de revisión integradora de literatura con marco temporal 2010-2020. Búsqueda realizada en cuatro bases de datos de acceso abierto. Estadística descriptiva utilizada para el cálculo de la prevalencia de geohelmintos y datos socioambientales. Se incluyó un total de 841,107 muestras humanas en los 55 estudios elegibles para esta revisión. La prevalencia combinada de geohelmintos en el país fue del 19\%. Ascaris lumbricoides (68\%) fue el más prevalente, seguido por anquilostomas (24\%) y Trichuris trichiura (8\%). Se observó una alta prevalencia en el Medio Oeste (22\%), Noreste (19\%) y Norte (14\%). El saneamiento deficiente, los hábitos de higiene inadecuados y los bajos ingresos fueron los factores asociados con la geohelmintiasis más reportados en los estudios. Las infecciones por geohelmintos todavía prevalecen en el país, aunque han disminuido ligeramente en los últimos cinco años. Entre los principales factores asociados a las infecciones se destacan las malas condiciones sanitarias, los hábitos higiénicos inadecuados y los bajos ingresos. Esperamos que los resultados de esta revisión sirvan de aporte a las políticas públicas y administraciones regionales con programas de sensibilización de la población sobre la prevención y atención para no propagar la infección, además de inversiones en infraestructura en el sector de recolección de basura, red de desagüe, tratamiento de aguas y ampliar la administración masiva de tratamientos antiparasitarios en Brasil.

Palabras clave: Helmintos; Ascaris lumbricoides; Trichuris trichiura; Anquilostomas.

\section{Introdução}

Os geo-helmintos (helmintos transmitidos pelo solo), estão entre as doenças tropicais negligenciadas, mais prevalentes com uma estimativa de 1,5 bilhões de pessoas infectadas com pelo menos uma espécie em regiões tropicais na África, Ásia e América Latina (WHO, 2020). Ascaris lumbricoides é o geo-helminto mais prevalente, infectando cerca de 1,2 bilhões de pessoas, seguido por Trichuris trichiura (infectando cerca de 795 milhões de pessoas) e Ancilostomídeos (Ancylostoma duodenale e Necator americanus), que infectam cerca de 740 milhões de pessoas em todo o mundo (Parija et al., 2017; WHO, 2020).

A alta prevalência de geo-helmintos nas regiões tropicais está associada ao baixo desenvolvimento socioeconômico e condições ambientais adequadas, temperaturas quentes e umidade, que favorecem a transmissão desses helmintos (Brooker et al., 2006). A infecção humana é influenciada pela pobreza, higiene pessoal inadequada, escassez de água potável, saneamento precário e superlotação (Gyorkos et al, 2014; Brooker et al., 2008). Evidências sugerem que o grupo mais vulnerável são crianças em razão da imaturidade imunológica e da inexperiência dos hábitos higiênicos, além de manter contato frequente com o solo (Brasil, 2018; Brooker et al., 2006; Galvani et al., 2005).

A infecção pelos geo-helmintos geralmente não resulta em mortalidade, mas leva a infecções crônicas e morbidade estendida. Podem resultar em anemia, desnutrição, retardo do crescimento e déficits cognitivos (Stoltzfus et al., 2001; Ostan et al., 2007).

Os métodos parasitológicos utilizados para o diagnóstico da infecção por geo-helmintos é baseado na observação microscópica dos ovos dos parasitas nas fezes. As técnicas mais indicadas e mais utilizadas para o levantamento das prevalências no Brasil são: método direto, sedimentação e kato-katz. Esse conjunto de métodos permanece como o instrumento mais largamente utilizado, principalmente por seu baixo custo operacional e sua praticidade em situações de infraestrutura laboratorial mais simples (Brasil, 2018). 
Segundo a Organização Mundial da Saúde (OMS), as principais estratégias de saúde pública para controlar a carga das geo-helmintíases em áreas endêmicas consiste no tratamento preventivo com a administração estratégica de medicamentos, o saneamento adequado, além do trabalho conjunto da vigilância em saúde e da atenção básica com ações de educação em saúde (WHO, 2012; 2020).

No Brasil, as geo-helmintíases estão amplamente distribuídas em todas as regiões, ocorrendo principalmente nas zonas rurais e periferias de centros urbanos, que se destacam pelo saneamento básico precário e pouco conhecimento sobre transmissão e prevenção (Brasil, 2018).

O objetivo deste estudo foi conhecer a prevalência e as condições socioambientais da população brasileira acometida por geo-helmintos nos últimos dez anos, através da análise de estudos epidemiológicos previamente publicados que abordam a prevalência de geo-helmintos e também os fatores de risco potenciais, identificando grupos-alvos e as regiões de alto risco que requerem maiores intervenções.

\section{Metodologia}

Trata-se de um estudo exploratório e descritivo de revisão integrativa de literatura embasada no método de Whittemore e Knafl (2005), que permite a síntese de múltiplos estudos, facilitando a análise das informações produzidas e conclusões gerais a respeito de uma área de estudo.

A revisão integrativa da literatura proporciona a reunião de achados a partir de diferentes métodos de pesquisa, permitindo a síntese dos resultados sem ferir os dados originais. Portanto, necessita ser realizada de maneira sistemática e rigorosa (Soares et al., 2014).

Este estudo seguiu as fases: formulação e identificação do problema de pesquisa, coleta de dados, avaliação dos dados, análise e interpretação dos dados coletados (síntese, exibição, comparação dos dados) e apresentação dos resultados da pesquisa.

\subsection{Problema de pesquisa}

Na primeira etapa, foi realizado um aprofundamento teórico sobre os geo-helmintos chegando-se à questão norteadora da pesquisa "Qual é a prevalência e os fatores socioambientais da população brasileira com geo-helmintíases nos últimos dez anos?".

\subsection{Coleta de dados}

Na segunda fase, realizou- se o levantamento bibliográfico através de buscas em 4 bases de dados: Biblioteca Virtual de Saúde (BVS), Base de dados bibliográficos do National of Medicine dos EUA (Medline), Literatura Latino-Americana e do Caribe em Ciências da Saúde (LILACS) e na Biblioteca Eletrônica Científica Online (SciELO). A busca ocorreu de novembro de 2020 a março de 2021, realizada por uma análise das palavras contidas no título, resumo e nos termos do índice utilizados para descrever os artigos.

Na primeira busca, para restringir a amostra, foi empregado o operador booleano AND, junto com os termos selecionados: Geo-helmintos AND Brasil. Na segunda busca, foi empregado o operador booleano OR junto com os termos: Ascaris lumbricoides OR Trichuris trichiura OR Ancilostomídeos OR Ancylostoma sp. OR Necator americanus OR helmintos OR geo-helmintos AND Brasil.

Por ser uma pesquisa limitada ao Brasil, a busca foi limitada aos idiomas Inglês, Espanhol e Português. Com critério cronológico e temporal estabelecido entre os anos 2010-2020. 
Vários métodos diagnósticos são disponíveis para a detecção de geo-helmintos tanto em pacientes como em comunidades saudáveis. No entanto, selecionamos estudos que usaram as abordagens diagnósticas convencionais através da microscopia, como os métodos com princípios de sedimentação, concentração, flutuação e adesivo.

\subsection{Avaliação dos dados}

$\mathrm{Na}$ terceira fase, os trabalhos encontrados foram analisados quanto à qualidade dos dados (consistência, clareza e assertividade das informações disponíveis) e se estavam relacionados ao problema de pesquisa.

\subsection{Análise dos dados}

A quarta fase, de análise dos dados, considerou as etapas de redução dos dados, tabulação, comparação e verificação. Os dados foram extraídos por meio de um instrumento próprio, contendo informações sobre autores, ano de publicação, bases de dados e periódicos, região do estudo, tamanho da amostra, tipo de população, sexo, faixa etária, dados socioambientais, métodos diagnósticos, prevalência total e espécies individuais de geo-helmintos.

A estatística descritiva foi utilizada para os cálculos dos percentuais de prevalências dos geo-helmintos e dados sociodemográficos com o programa Stata versão 13.1 (Stata Corp; College Station, EUA). A prevalência de cada geo-helminto foi calculada dividindo-se o número de sujeitos positivos para geo-helmintíases pelo total de participantes do estudo.

\subsection{Síntese e apresentação dos resultados}

Na quinta e última fase, foram apresentadas conclusões da revisão integrativa, juntamente com reflexões dos dados.

Não houve a necessidade de submissão ao Comitê de Ética em Pesquisa (CEP), pois trata-se de uma pesquisa bibliográfica na qual as fontes são de domínio público.

\section{Resultados e Discussão}

O resultado obtido a partir dos critérios de inclusão e parâmetros de busca, seleção e exclusão de artigos sobre a temática estão apresentados no fluxograma (Figura 1). Resultou em 956 artigos relacionados às estratégias de buscas empregadas que após os critérios de elegibilidade, 55 estudos permaneceram de base para esta revisão (Tabela 1). 
Figura 1. Fluxograma das etapas realizadas na revisão.

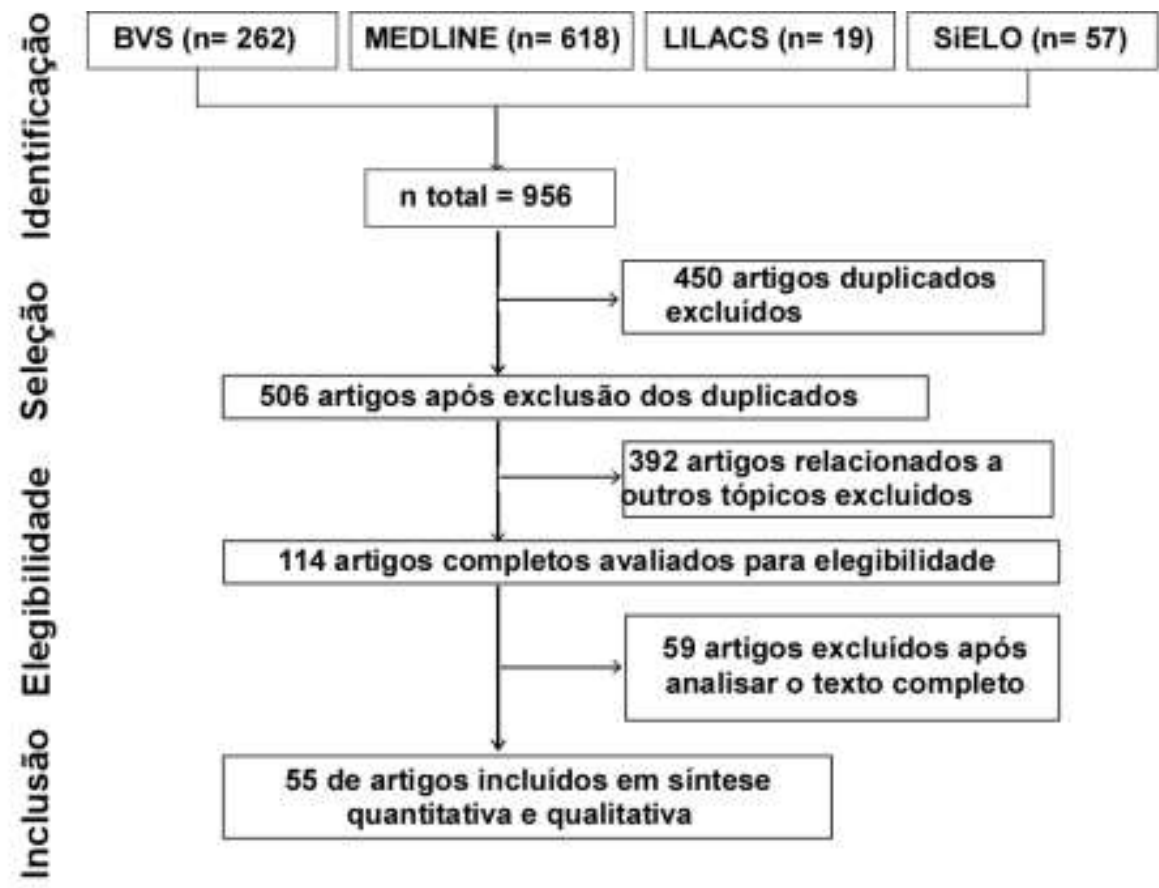

Fonte: Autores (2021).

Neste fluxograma é possível observar o número de estudos identificados nas quatro bases de dados de acesso aberto, utilizando os termos de busca detalhados na metodologia. Apresenta ainda, a sequência de artigos que permaneceram após os critérios de seleção e elegibilidade (textos duplicados, artigos relacionados com outros tópicos, textos excluídos pelos critérios da revisão) e o número final de artigos incluídos na revisão.

Tabela 1. Resumo dos estudos incluídos na revisão.

\begin{tabular}{|c|c|c|c|c|c|c|c|c|}
\hline Autor/ano & $\begin{array}{l}\text { Área do } \\
\text { estudo }\end{array}$ & $\begin{array}{c}\text { n } \\
\text { amost } \\
\text { ral }\end{array}$ & $\begin{array}{c}\% \\
\text { prevalência } \\
\text { de geo- } \\
\text { helmintos }\end{array}$ & $\begin{array}{c}\% \\
\text { Ascaris } \\
\text { lumbric } \\
\text { oides }\end{array}$ & $\begin{array}{c}\% \\
\text { Trichuris } \\
\text { trichiura }\end{array}$ & $\begin{array}{c}\% \\
\text { Ancilostom } \\
\text { ídeos }\end{array}$ & $\begin{array}{c}\text { Método } \\
\text { diagnóstico }\end{array}$ & $\begin{array}{c}\text { Dados } \\
\text { socioambientais } \\
\text { associados às } \\
\text { infecções }\end{array}$ \\
\hline $\begin{array}{l}\text { ALMEIDA et } \\
\text { al., } 2020\end{array}$ & MA & 259 & 24 & 39 & 0 & 61 & $\begin{array}{l}\text { Hoffman, Pons } \\
\text { e Janer; Willis }\end{array}$ & $\begin{array}{c}\text { Baixo nível } \\
\text { socioenômico - renda }\end{array}$ \\
\hline $\begin{array}{l}\text { ANTUNES et } \\
\text { al., } 2020\end{array}$ & GO & 43 & 18 & 88 & 0 & 12 & $\begin{array}{l}\text { Hoffman, Pons } \\
\text { e Janer; } \\
\text { Baermann e } \\
\text { Moraes }\end{array}$ & $\begin{array}{l}\text { Hábitos inadequados } \\
\text { de higiene; } \\
\text { Saneamento precário }\end{array}$ \\
\hline $\begin{array}{l}\text { ALENCAR et } \\
\text { al., } 2020\end{array}$ & MT & 53 & 4 & 100 & 0 & 0 & $\begin{array}{l}\text { Hoffman, Pons } \\
\text { e Janer; } \\
\text { Sheather }\end{array}$ & $\begin{array}{l}\text { Ausência de exame de } \\
\text { rotina e tratamento }\end{array}$ \\
\hline $\begin{array}{l}\text { CHAVES et } \\
\text { al., } 2020\end{array}$ & PB & 69 & 6 & 75 & 25 & 0 & $\begin{array}{l}\text { Hoffman, Pons } \\
\text { e Janer }\end{array}$ & $\begin{array}{c}\text { Baixo nível } \\
\text { socioeconômico - } \\
\text { escolaridade - renda; } \\
\text { Saneamento precário }\end{array}$ \\
\hline $\begin{array}{l}\text { EUSTACHIO } \\
\text { et al., } 2019\end{array}$ & MG & 66 & 15 & 70 & 10 & 20 & $\begin{array}{l}\text { Hoffman, Pons } \\
\text { e Janer }\end{array}$ & $\begin{array}{l}\text { Saneamento precário; } \\
\text { Ausência de exame de } \\
\text { rotina e tratamento }\end{array}$ \\
\hline $\begin{array}{l}\text { OISHI et al., } \\
2019\end{array}$ & PR & 549 & 3 & 72 & 28 & 0 & Ritchie & ni \\
\hline $\begin{array}{l}\text { REIS et al., } \\
2019\end{array}$ & BA & 144 & 63 & 15 & 9 & 76 & $\begin{array}{l}\text { Baermann e } \\
\text { Moraes; Kato- } \\
\text { Katz; Graham }\end{array}$ & $\begin{array}{c}\text { Baixo nível } \\
\text { socioeconômico - } \\
\text { escolaridade - renda }\end{array}$ \\
\hline $\begin{array}{l}\text { SILVA et al., } \\
2019\end{array}$ & MS & 200 & 12 & 36 & 64 & 0 & $\begin{array}{l}\text { Hoffman, Pons } \\
\text { e Janer }\end{array}$ & $\begin{array}{l}\text { Hábitos inadequados } \\
\text { de higiene; }\end{array}$ \\
\hline
\end{tabular}




\begin{tabular}{|c|c|c|c|c|c|c|c|c|}
\hline $\begin{array}{l}\text { SILVA, } \\
\text { ROCHA, } \\
2019\end{array}$ & PE & 477 & 36 & 53 & 41 & 6 & Ritchie & $\begin{array}{c}\text { Saneamento precário } \\
\text { ni }\end{array}$ \\
\hline $\begin{array}{l}\text { SEGUÍ et al., } \\
2018\end{array}$ & PR & 766 & 11 & 47 & 43 & 10 & Kato-Katz & ni \\
\hline $\begin{array}{l}\text { ANDRADE et } \\
\text { al., } 2018\end{array}$ & BA & 144 & 63 & 15 & 9 & 76 & $\begin{array}{l}\text { Baermann e } \\
\text { Moraes; Kato- } \\
\text { Katz }\end{array}$ & $\begin{array}{c}\text { Má qualidade da } \\
\text { água; Hábitos } \\
\text { inadequados de } \\
\text { higiene; Saneamento } \\
\text { precário }\end{array}$ \\
\hline $\begin{array}{l}\text { BARBOSA et } \\
\text { al., } 2018\end{array}$ & $\mathrm{RJ}$ & 294 & 9 & 8 & 11 & 81 & $\begin{array}{l}\text { Hoffman, Pons } \\
\text { e Janer }\end{array}$ & Área rural \\
\hline $\begin{array}{l}\text { IASBIK et al., } \\
2018\end{array}$ & MG & 419 & 14 & 58 & 8 & 34 & $\begin{array}{l}\text { Hoffman, Pons } \\
\text { e Janer }\end{array}$ & $\begin{array}{c}\text { Saneamento precário; } \\
\text { Ausência de } \\
\text { programas de } \\
\text { educação em saúde }\end{array}$ \\
\hline $\begin{array}{l}\text { JESKE et al., } \\
2018\end{array}$ & $\mathrm{RS}$ & 73 & 36 & 92 & 8 & 0 & Faust; Ritchie & $\begin{array}{l}\text { Ausência de exame de } \\
\text { rotina e tratamento }\end{array}$ \\
\hline $\begin{array}{l}\text { SILVA et al, } \\
2018 \mathrm{a}\end{array}$ & MT & 183 & 25 & 57 & 43 & 0 & $\begin{array}{l}\text { Hoffman, Pons } \\
\text { e Janer }\end{array}$ & Saneamento precário \\
\hline $\begin{array}{l}\text { SILVA et al., } \\
2018 b\end{array}$ & MS & 196 & 25 & 2 & 27 & 71 & $\begin{array}{l}\text { Hoffman, Pons } \\
\text { e Janer }\end{array}$ & $\begin{array}{l}\text { Hábitos inadequados } \\
\text { de higiene; } \\
\text { Saneamento precário }\end{array}$ \\
\hline $\begin{array}{l}\text { BANHOS et } \\
\text { al., } 2017\end{array}$ & PA & 367 & 13 & 70 & 17 & 13 & $\begin{array}{l}\text { Hoffman, Pons } \\
\text { e Janer }\end{array}$ & $\begin{array}{l}\text { Má qualidade da } \\
\text { água; Saneamento } \\
\text { precário; Baixo nível } \\
\text { socioenômico - renda }\end{array}$ \\
\hline $\begin{array}{l}\text { FARIA et al., } \\
2017\end{array}$ & $\mathrm{RJ}$ & 3245 & 1 & 36 & 30 & 34 & $\begin{array}{l}\text { Baermann e } \\
\text { Moraes; Kato- } \\
\text { Katz; Graham }\end{array}$ & $\begin{array}{c}\text { Baixo nível } \\
\text { socioenômico - } \\
\text { escolaridade e renda }\end{array}$ \\
\hline $\begin{array}{l}\text { FONSECA et } \\
\text { al., } 2017\end{array}$ & SP & 576 & 23 & 78 & 13 & 9 & TF - Test & $\begin{array}{l}\text { Má qualidade da } \\
\text { água; Ausência de } \\
\text { programas de } \\
\text { educação em saúde }\end{array}$ \\
\hline $\begin{array}{l}\text { IGNÁCIO et } \\
\text { al., } 2017\end{array}$ & $\mathrm{RJ}$ & 595 & 3 & 37 & 26 & 37 & $\begin{array}{l}\text { Hoffman, Pons } \\
\text { e Janer }\end{array}$ & $\begin{array}{l}\text { Hábitos inadequados } \\
\text { de higiene; } \\
\text { Saneamento precário }\end{array}$ \\
\hline $\begin{array}{l}\text { MOURA et } \\
\text { al., } 2017\end{array}$ & $\mathrm{RS}$ & 140 & 6 & 37 & 13 & 50 & $\begin{array}{c}\text { Ritchie; } \\
\text { Hoffman, Pons } \\
\text { e Janer; } \\
\text { Baermann e } \\
\text { Moraes; Willis; } \\
\text { Ziehl-Neelsen }\end{array}$ & $\begin{array}{l}\text { Hábitos inadequados } \\
\text { de higiene }\end{array}$ \\
\hline $\begin{array}{l}\text { NAGEL et al., } \\
2017\end{array}$ & RS & 209 & 20 & 97 & 3 & 0 & $\begin{array}{l}\text { Hoffman, Pons } \\
\text { e Janer; Faust; } \\
\text { Ritchie }\end{array}$ & $\begin{array}{c}\text { Ausência de } \\
\text { programas de } \\
\text { educação em saúde }\end{array}$ \\
\hline $\begin{array}{l}\text { CASAVECHI } \\
\text { A et al., } 2016\end{array}$ & PR & 775 & 1 & 0 & 20 & 80 & $\begin{array}{l}\text { Hoffman, Pons } \\
\text { e Janer; Faust; } \\
\text { Rugai }\end{array}$ & $\mathrm{ni}$ \\
\hline $\begin{array}{l}\text { DAMÁZIO et } \\
\text { al., } 2016\end{array}$ & ES & 221 & 17 & 16 & 13 & 71 & $\begin{array}{l}\text { Hoffman, Pons } \\
\text { e Janer }\end{array}$ & $\begin{array}{c}\text { Ausência de } \\
\text { programas de } \\
\text { educação em saúde }\end{array}$ \\
\hline $\begin{array}{l}\text { BIOLCHI et } \\
\text { al., } 2015\end{array}$ & $\mathrm{SC}$ & 109 & 37 & 45 & 3 & 52 & $\begin{array}{l}\text { Hoffman, Pons } \\
\text { e Janer }\end{array}$ & $\begin{array}{c}\text { Saneamento precário; } \\
\text { Área rural }\end{array}$ \\
\hline $\begin{array}{l}\text { PEREIRA et } \\
\text { al., } 2015\end{array}$ & $\mathrm{DF}$ & 75 & 16 & 58 & 0 & 42 & $\begin{array}{l}\text { Hoffman, Pons } \\
\text { e Janer; Rugai }\end{array}$ & $\begin{array}{c}\text { Hábitos inadequados } \\
\text { de higiene }\end{array}$ \\
\hline $\begin{array}{l}\text { ROLLEMBE } \\
\text { RG et al., } \\
2015\end{array}$ & SE & 500 & 82 & 36 & 43 & 21 & Kato-katz & $\begin{array}{c}\text { Má qualidade da } \\
\text { água; Saneamento } \\
\text { precário }\end{array}$ \\
\hline $\begin{array}{l}\text { BRAGAGNO } \\
\text { LI, } \\
2014\end{array}$ & PB & 1004 & 43 & 60 & 39 & 1 & Ritchie & $\begin{array}{c}\text { Baixo nível } \\
\text { socioenômico - } \\
\text { escolaridade e renda }\end{array}$ \\
\hline $\begin{array}{l}\text { COUTO et al., } \\
2014\end{array}$ & MG & 503 & 0,19 & 0 & 100 & 0 & $\begin{array}{l}\text { Kato-katz; } \\
\text { Formol-eter }\end{array}$ & ni \\
\hline $\begin{array}{l}\text { FERNANDE } \\
\mathrm{S} \text { et al., } 2014\end{array}$ & PI & 251 & 13 & 72 & 0 & 28 & $\begin{array}{l}\text { Hoffman, Pons } \\
\text { e Janer; Willis }\end{array}$ & $\begin{array}{c}\text { Hábitos inadequados } \\
\text { de higiene }\end{array}$ \\
\hline FERRAZ et & MA & 405 & 43 & 67 & 19 & 14 & Hoffman, Pons & Saneamento precário; \\
\hline
\end{tabular}




\begin{tabular}{|c|c|c|c|c|c|c|c|}
\hline al., 2014 & $\mathrm{AP}$ & 9.842 & 3 & 76 & 11 & 13 & e Janer \\
\hline $\begin{array}{l}\text { MALHEIROS } \\
\text { et al., } 2014\end{array}$ & MT & 542 & 19 & 0 & 0 & 100 & $\begin{array}{c}\text { Faust; Formol- } \\
\text { eter }\end{array}$ \\
\hline $\begin{array}{l}\text { NERES- } \\
\text { NORBERG et } \\
\text { al., } 2014\end{array}$ & MS & 134 & 57 & 57 & 31 & 12 & $\begin{array}{l}\text { Hoffman, Pons } \\
\text { e Janer; Willis }\end{array}$ \\
\hline $\begin{array}{l}\text { DAMÁZIO et } \\
\text { al., } 2013\end{array}$ & ES & 82 & 17 & 7 & 7 & 86 & $\begin{array}{c}\text { Hoffman, Pons } \\
\text { e Janer }\end{array}$ \\
\hline $\begin{array}{l}\text { AGUIAR- } \\
\text { SANTOS et } \\
\text { al., } 2013\end{array}$ & PE & 102 & 73 & 45 & 30 & 25 & $\begin{array}{c}\text { Hoffman, Pons } \\
\text { e Janer }\end{array}$ \\
\hline $\begin{array}{l}\text { ASSIS et al., } \\
2013\end{array}$ & MG & 409 & 43 & 11 & 1 & 88 & TF-Test \\
\hline $\begin{array}{l}\text { MATA- } \\
\text { SANTOS et } \\
\text { al., } 2013\end{array}$ & RS & 144 & 17 & 64 & 36 & 0 & $\begin{array}{c}\text { Hoffman, Pons } \\
\text { e Janer }\end{array}$ \\
\hline $\begin{array}{l}\text { SANTOS et } \\
\text { al., 2013a }\end{array}$ & $\mathrm{BA}$ & $\begin{array}{c}374.1 \\
20\end{array}$ & 0,27 & 0 & 0 & 100 & $\begin{array}{c}\text { Baermann e } \\
\text { Moraes }\end{array}$ \\
\hline $\begin{array}{l}\text { SANTOS et } \\
\text { al., 2013b }\end{array}$ & $\mathrm{BA}$ & 97 & 47 & 61 & 22 & 17 & Ritchie \\
\hline $\begin{array}{l}\text { ALMEIDA et } \\
\text { al., } 2012\end{array}$ & BA & 349 & 45 & 3 & 0 & 97 & $\begin{array}{c}\text { Hoffman, Pons } \\
\text { e Janer }\end{array}$ \\
\hline
\end{tabular}

\begin{tabular}{|c|c|c|c|c|c|c|c|c|}
\hline $\begin{array}{l}\text { BRANCO et } \\
\text { al., } 2012\end{array}$ & $\mathrm{SP}$ & 185 & 25 & 58 & 38 & 4 & Kato-katz & $\begin{array}{l}\text { educação em saúde } \\
\text { Saneamento precário; } \\
\text { Ausência de } \\
\text { programas de } \\
\text { educação em saúde }\end{array}$ \\
\hline $\begin{array}{l}\text { BARBOSA et } \\
\text { al., } 2012\end{array}$ & PE & $\begin{array}{c}11.23 \\
4\end{array}$ & 80 & 44 & 45 & 11 & Kato-katz & ni \\
\hline $\begin{array}{l}\text { BRANDELLI } \\
\text { et al., } 2012\end{array}$ & RS & 62 & 48 & 67 & 20 & 13 & $\begin{array}{l}\text { Hoffman, Pons } \\
\text { e Janer; Kato- } \\
\text { katz; Faust }\end{array}$ & $\begin{array}{c}\text { Hábitos inadequados } \\
\text { de higiene; } \\
\text { Saneamento precário }\end{array}$ \\
\hline $\begin{array}{l}\text { MORTEAN } \\
\text { et al., } 2012\end{array}$ & PR & 5219 & 2 & 47 & 29 & 24 & $\begin{array}{l}\text { Hoffman, Pons } \\
\text { e Janer; Faust; } \\
\text { Baermann e } \\
\text { Moraes }\end{array}$ & ni \\
\hline $\begin{array}{l}\text { SILVA et al., } \\
2012\end{array}$ & $\mathrm{AM}$ & 65 & 86 & 62 & 20 & 18 & $\begin{array}{l}\text { Hoffman, Pons } \\
\text { e Janer }\end{array}$ & $\begin{array}{c}\text { Ausência de } \\
\text { programas de } \\
\text { educação em saúde; } \\
\text { Ausência de exame de } \\
\text { rotina e tratamento }\end{array}$ \\
\hline $\begin{array}{l}\text { BELLOTO et } \\
\text { al., } 2011\end{array}$ & SP & 310 & 4 & 100 & 0 & 0 & $\begin{array}{c}\text { Hoffman, Pons } \\
\text { e Janer; Faust }\end{array}$ & $\begin{array}{c}\text { Saneamento precário; } \\
\text { Ausência de } \\
\text { programas de } \\
\text { educação em saúde }\end{array}$ \\
\hline $\begin{array}{l}\text { ROLLEMBE } \\
\text { RG et al., } \\
2011\end{array}$ & $\mathrm{SE}$ & $\begin{array}{c}417.0 \\
39\end{array}$ & 34 & 71 & 4 & 25 & Kato-katz & $\begin{array}{l}\text { Baixo nível } \\
\text { socioenômico - } \\
\text { escolaridade }\end{array}$ \\
\hline $\begin{array}{l}\text { SEIXAS et } \\
\text { al., } 2011\end{array}$ & $\mathrm{BA}$ & 200 & 37 & 68 & 28 & 4 & $\begin{array}{l}\text { Hoffman, Pons } \\
\text { e Janer }\end{array}$ & $\begin{array}{l}\text { Baixo nível } \\
\text { socioenômico-- } \\
\text { renda; Ausência de } \\
\text { programas de } \\
\text { educação em saúde }\end{array}$ \\
\hline $\begin{array}{l}\text { SILVA et al., } \\
2011\end{array}$ & MA & 220 & 54 & 100 & 0 & 0 & $\begin{array}{l}\text { Hoffman, Pons } \\
\text { e Janer }\end{array}$ & Saneamento precário \\
\hline $\begin{array}{l}\text { VALVERDE } \\
\text { et al., } 2011\end{array}$ & $\mathrm{AM}$ & 463 & 58 & 45 & 39 & 16 & $\begin{array}{c}\text { Ritchie; } \\
\text { Baermann e }\end{array}$ & $\begin{array}{l}\text { Ausência de exame de } \\
\text { rotina e tratamento }\end{array}$ \\
\hline
\end{tabular}




\begin{tabular}{|c|c|c|c|c|c|c|c|c|}
\hline & & & & & & & $\begin{array}{l}\text { Moraes; } \\
\text { Graham }\end{array}$ & \\
\hline $\begin{array}{l}\text { VISSER et al., } \\
2011\end{array}$ & $\mathrm{AM}$ & 362 & 10 & 74 & 18 & 8 & $\begin{array}{l}\text { Hoffman, Pons } \\
\text { e Janer }\end{array}$ & $\begin{array}{l}\text { Má qualidade da } \\
\text { água; Infraestrutura } \\
\text { sanitária precária }\end{array}$ \\
\hline $\begin{array}{l}\text { OLIVEIRA et } \\
\text { al., } 2010\end{array}$ & $\mathrm{AM}$ & 400 & 26 & 46 & 17 & 37 & $\begin{array}{l}\text { Hoffman, Pons } \\
\text { e Janer }\end{array}$ & $\begin{array}{c}\text { Baixo nível } \\
\text { socioenômico - } \\
\text { renda; Saneamento } \\
\text { precário }\end{array}$ \\
\hline $\begin{array}{l}\text { SILVA et al., } \\
2010\end{array}$ & MA & 3933 & 4 & 75 & 0 & 25 & $\begin{array}{l}\text { Hoffman, Pons } \\
\text { e Janer }\end{array}$ & ni \\
\hline $\begin{array}{l}\text { FONSECA et } \\
\text { al., } 2010\end{array}$ & $\mathrm{PA}$ & 2523 & 52 & 48 & 23 & 29 & Kato-katz & $\begin{array}{c}\text { Baixo nível } \\
\text { socioenômico - } \\
\text { escolaridade e renda }\end{array}$ \\
\hline $\begin{array}{l}\text { SILVA, } \\
\text { SILVA, } 2010\end{array}$ & MG & 161 & 72 & 70 & 0 & 30 & $\begin{array}{l}\text { Hoffman, Pons } \\
\text { e Janer; } \\
\text { Baermann e } \\
\text { Moraes }\end{array}$ & Saneamento precário \\
\hline
\end{tabular}

Abreviações: ni - não identificado; MA - Maranhão; GO - Goiás; MT - Mato Grosso; PB - Paraíba; MG - Minas Gerais; MS - Mato Grosso do Sul; PE - Pernambuco; PR - Paraná; BA - Bahia; RJ - Rio de Janeiro; RS - Rio Grande do Sul; PA - Pará; SP - São Paulo; ES - Espírito Santo; SC - Santa Catarina; SE - Sergipe; PI - Piauí; AM - Amazonas. Fonte: Autores (2021)..

Na Tabela 1 observamos em detalhes os artigos incluídos na revisão contendo as informações extraídas necessárias para as análises, como autores, ano de publicação, região do estudo, tamanho da amostra, dados socioambientais, métodos diagnósticos, prevalência total e espécies individuais de geo-helmintos.

Em relação ao número de artigos acerca da infecção por geo-helmintos na população brasileira ao longo dos anos, os maiores picos de publicações foram atingidos entre os anos de 2011 e 2014 (6 publicações/ano), 2017 (6), 2018 (7) e 2019 (5), enquanto que nos outros anos foram publicados de 2 a 4 artigos, sendo 2010 (4), 2015 (3), 2016 (2) e 2020 (4). Esses dados sugerem que as publicações científicas na área de infecção humana por geo-helmintos é estável e sem tendência de aumento no Brasil, fato que deveria ser mudado para os próximos anos já que estudos dessa natureza são indicadores da ausência de saneamento básico tornando-os dados importantes para o aprimoramento das políticas públicas de saúde.

Foi encontrado maior número de estudos realizados nas regiões Sudeste e Nordeste, correspondendo a aproximadamente $23 \%$ (13/55) e 25\% (14/55) do total de estudos, respectivamente. A região Norte apresentou 20\% (11/50), Sul com 18\% (10/50) e com menor número de estudos a região Centro-Oeste, com aproximadamente 14\% (8/55) do total. Esse resultado reflete uma amostragem das cinco regiões brasileiras, contudo as regiões Sudeste e Nordeste estão melhor caracterizadas apresentando maiores produções científicas, embora ainda existam lacunas em muitos estados brasileiros que não apresentaram nenhum dado sobre geo-helmintíases na população. Assim, estudos sobre a detecção de geo-helmintos nessas regiões devem ser incentivados.

Doze métodos parasitológicos individualmente ou em combinação foram usados nos artigos incluídos nessa revisão. O esfregaço fecal espesso de Kato-Katz é o método parasitológico recomendado pela Organização Mundial da Saúde para a detecção de geo-hlemintos (WHO, 1994; 2013). No entanto, esta revisão indicou que o método baseado em sedimentação espontânea descrito por Hoffman, Pons e Janer (HPJ), também conhecido como Lutz foi o mais utilizado nos trabalhos realizados no Brasil (43\%), seguido da técnica de Kato-Katz aplicada em 13\% dos trabalhos (Tabela 2). É notável a preferência da técnica de Sedimentação Espontânea de Hoffmann, Pons e Janner utilizada na maioria dos trabalhos realizados no país. A preferência por essa técnica pode ser justificada pela sua praticidade, sensibilidade e reprodutibilidade nos laboratórios de pesquisa, já que nenhum reagente é utilizado, deixando-a uma técnica de baixo custo (Neves, 2016).

Outras técnicas de detecção como Baermann e Moraes foram utilizadas em 10\% dos estudos incluídos na revisão, Ritchie em 9\%, Faust em 8\%, Willis em 5\%, Graham em 4\%, TF Test (Three Fecal Test) em 2\%, Formol-eter em 2\%, Rugai em $2 \%$, Sheather em $1 \%$ e Ziehl-Neelsen também em $1 \%$. Vale ressaltar que na maioria dos estudos, mais de uma técnica foi 
utilizada. A associação de técnicas geralmente garante melhor resultado e, portanto, maior confiabilidade, principalmente quando se utilizam técnicas de fundamentos diferentes (Periago et al., 2015).

Tabela 2. Técnicas parasitológicas utilizadas para detecção de geo-helmintos nas amostras humanas no Brasil.

\begin{tabular}{llcc}
\hline \multicolumn{1}{c}{ Técnica } & \multicolumn{1}{c}{ Princípio } & Número de estudos & $\%$ \\
\hline Hoffman, Pons e Janer & Sedimentação & 36 & 43 \\
Kato-Katz & Semi-concentração & 11 & 13 \\
Baermann e Moraes & Concentração & 9 & 10 \\
Ritchie & Centrífugo-sedimentação & 8 & 9 \\
Faust & Centrifugo-flutuação & 7 & 8 \\
Willis & Flutuação & 4 & 5 \\
Graham & Adesivo & 3 & 4 \\
TF Test & Concentração & 2 & 2 \\
Formol-eter & Centrífugo-flutuação & 2 & 2 \\
Rugai & Concentração & 2 & 2 \\
Sheather & Flutuação & 1 & 1 \\
Ziehl-Neelsen & Coloração & 1 & 1 \\
\hline
\end{tabular}

Fonte: Autores (2021).

Na Tabela 2 estão destacadas todas as técnicas parasitológicas utilizadas para identificação dos geo-helmintos reportadas nos artigos analisados nesta revisão, apresentando os valores absolutos e relativos de cada técnica.

\subsection{Prevalência de geo-helmintos na população brasileira}

Nos 55 estudos que analisaram amostras fecais humanas, foram realizados 841.107 testes coprológicos, os quais incluíram amostras de 19 estados brasileiros e do Distrito Federal. A prevalência de geo-helmintos reportada nos estudos analisados estava compreendida na margem de $0,19 \%$ a $86 \%$. Quando analisada a prevalência combinada dos estudos selecionados, encontramos a prevalência geral de geo-helmintos na população brasileira de 19\%. Esse resultado é de grande importância já que compilou um número expressivo de amostras e mostrou que o quadro geral das geo-helmintíases no Brasil nos últimos dez anos é expressivo.

Existem diferenças claras na prevalência combinada de geo-helmintos entre as regiões do Brasil. A maior prevalência foi observada na região Centro-Oeste, enquanto a baixa prevalência combinada foi observada na região Sul (Figura 2A). Isso pode estar associado a fatores ambientais e climáticos (temperatura, precipitação e umidade) que contribuem para a alta prevalência de geo-helmintos nas regiões Centro-Oeste, Nordeste e Norte. O Brasil é um país extenso e apresenta muitas diferenças regionais e intrarregionais nas condições de desenvolvimento socioeconômicos e sanitários. Segundo o Sistema Nacional de Informações sobre o Saneamento - SNIS, na região Norte apenas 12,3\% da população tem acesso a esgoto e $22 \%$ do esgoto coletado é tratado, no Nordeste apenas $28,5 \%$ tem acesso a esgoto e $33,7 \%$ é tratado, no Centro-Oeste $57,7 \%$ tem acesso a esgoto e 56,8\% é tratado, no Sudeste $79,21 \%$ tem acesso a esgoto e desses 55,5\% é tratado e no Sul 46,3\% da população tem acesso a esgoto e $47 \%$ do esgoto coletado é tratado (Brasil, 2019). Em locais com serviços de saneamento deficientes, as doenças podem ocorrer devido à contaminação de águas residuais que escoam a céu aberto em valas nas ruas ou são despejadas sem coleta e tratamento, contaminando o solo e rios. 
Analisando a prevalência geral de geo-helmintos específica por estados, Pernambuco (78\%), Pará (47\%), Paraíba (41\%), Santa Catarina (37\%), Amazonas (36\%), e Sergipe (34\%) apresentaram as maiores prevalências, os demais estados apresentaram menores porcentagens, conforme observado na Figura 2B.

É possível observar nos resultados dessa pesquisa o contraste das prevalências de geo-helmintos entre as regiões do Brasil, bem como grandes diferenças de prevalências entre os estados de uma mesma região, como no Norte em que o Amapá apresentou prevalência de 3\% e Pará e Amazonas com 47\% e 36\%, respectivamente. O mesmo é observado nos estados do Nordeste, Sudeste e Sul. Os estados da região Centro-Oeste apresentaram prevalências similares, entre $16 \%$ a $28 \%$. Devido às geo-helmintíases estarem fortemente associadas ao nível socioeconômico e ambiental da população, este dado epidemiológico pode ser utilizado como uma ferramenta na identificação de áreas de vulnerabilidade social.

Figura 2. Mapa da prevalência geral de geo-helmintos distribuído por regiões e estados entre o total de artigos analisados.

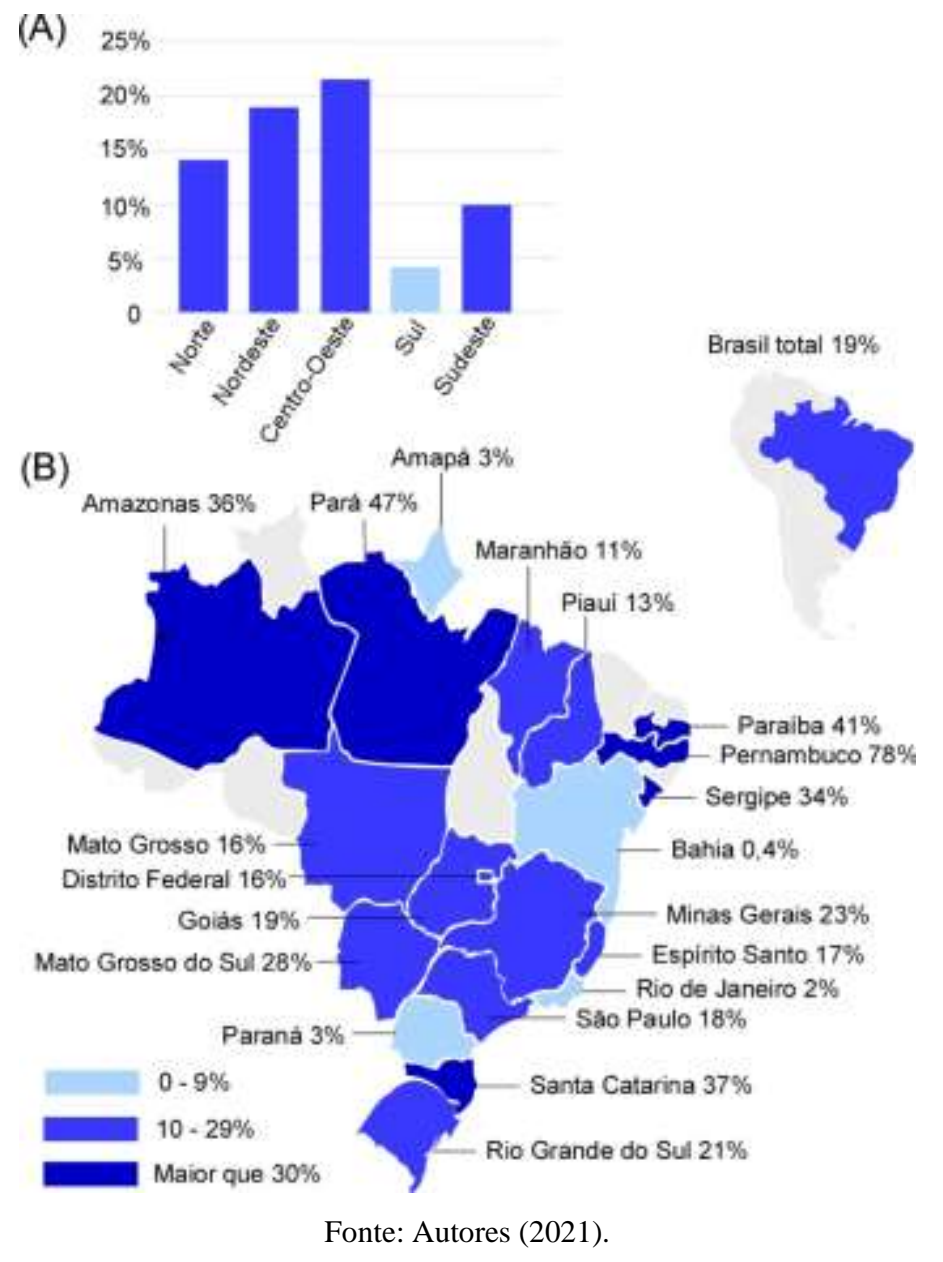

A Figura 2A apresenta com diferentes intensidades de cor, as porcentagens de prevalência combinada de geohelmintos separadas por região geográfica. A Figura 2B apresenta as porcentagens combinadas de prevalências identificadas nos estados brasileiros. Esse mapa possibilita uma ideia do cenário de distribuição dos geo-helmintos na população brasileira.

Os geo-helmintos identificados nas amostras humanas foram Ascaris lumbricoides, Trichuris trichiura e Ancilostomídeos. As análises revelaram que A. lumbricoides foi o geo-helminto mais prevalente encontrado nos estudos com 68\%, seguido de Ancilostomídeos (24\%) e T. trichiura (8\%) (Figura 3A), o que está de acordo com a tendência global, relatadas em meta-análises realizadas na Etiópia (Hailegebriel et al., 2020), Nigéria (Karshima, 2018), América do Sul (Chammartin et al., 2013) e Sudeste da Ásia (Silver et al., 2018). 
A maior prevalência de A. lumbricoides está geralmente associada à adequação das condições ambientais juntamente com a alta fecundidade dessa espécie e a durabilidade de seus ovos em solo contaminado, podendo esse geo-helminto adulto produzir até 200.000 ovos por dia (Neves et al., 2016; Rey et al, 2018).

A prevalência de A. lumbricoides, T. trichiura e Ancilostomídeos variou de região para região no Brasil. A maior prevalência de A. lumbricoides foi observada nas regiões Norte (53\%), Nordeste (44\%) e Sul (60\%), enquanto que os Ancilostomídeos foram mais prevalentes nas regiões Centro-Oeste (67\%) e Sudeste (49\%) (Figura 3B).

Figura 3. Prevalência das espécies de geo-helmintos relatados nos estudos no Brasil.

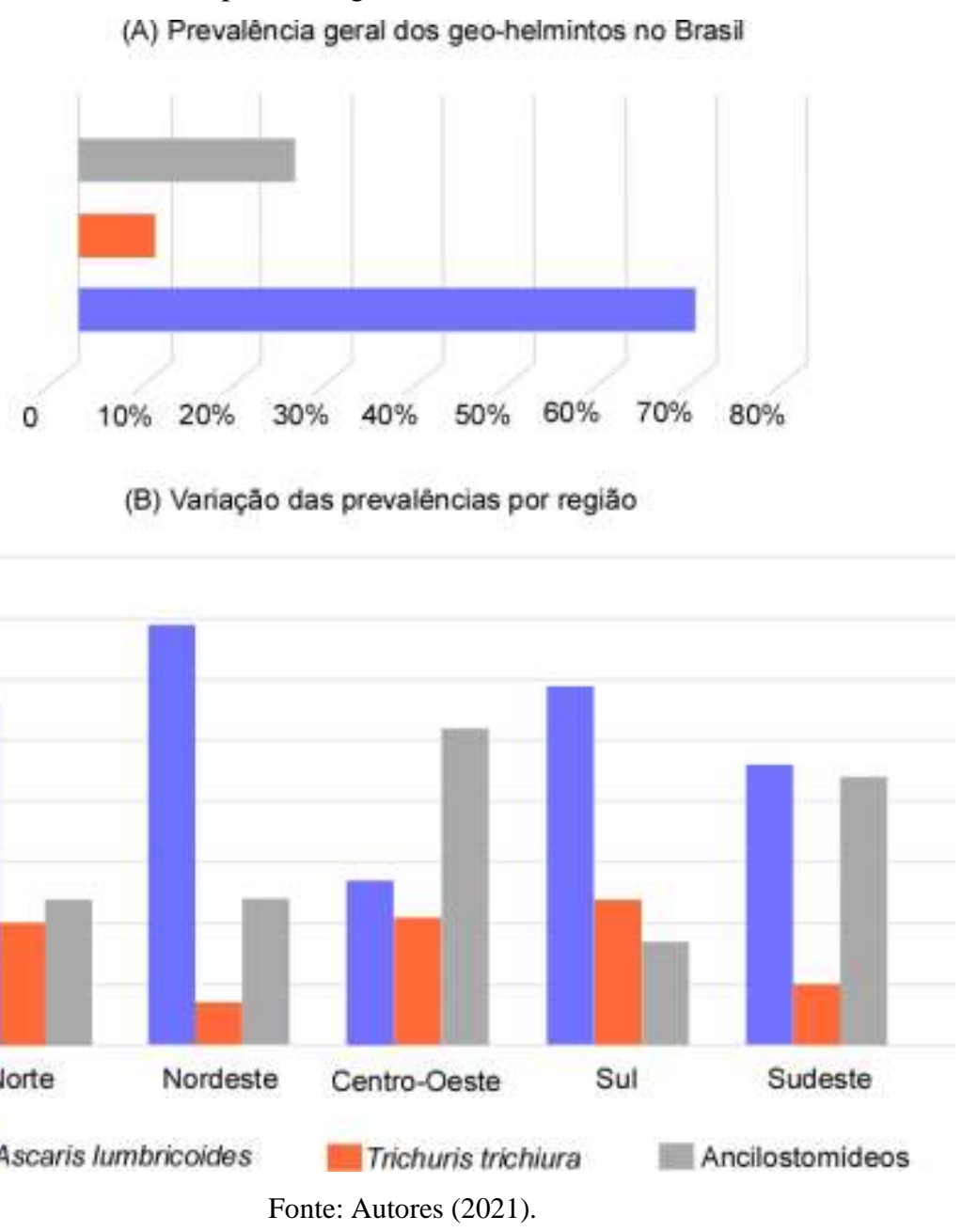

A Figura 3 ilustra a prevalência combinada por espécies de geo-helmintos identificados nos artigos incluídos nesta revisão e como cada espécie está distribuída nas diferentes regiões brasileiras.

A prevalência combinada de geo-helmintos entre os anos de 2010 a 2015 e 2016 a 2020 foi de $19 \%$ e $10 \%$, respectivamente (Figura 4A). A Figura 4B mostra a variação da prevalência combinada por geo-helminto, A. lumbricoides, $T$. trichiura e Ancilostomídeos através dos anos. Estas análises sugerem a tendência de declínio na prevalência de geo-helmintos na população brasileira que diminuiu nos últimos cinco anos em quase $10 \%$. As prevalências mais altas foram observadas antes de 2015 com picos em 2012 e 2015, enquanto que as prevalências mais baixas foram observadas a partir de 2015, com destaque em 2016 e 2017. Esse declínio pode ser o reflexo, embora tardio, da resolução WHA54.19 aprovada na 54 Assembléia Mundial de Saúde, realizada em 2001, que solicitou aos países membros endêmicos, entre eles o Brasil, que realizassem intervenções para o controle das geo-helmintíases (Brasil, 2018). 
Figura 4. Dados da variação na prevalência de geo-helmintos na população brasileira nos últimos 10 anos.

(A) Variação da prevalēncia geral de geo-helmintos entre os anos 2010-2015 e

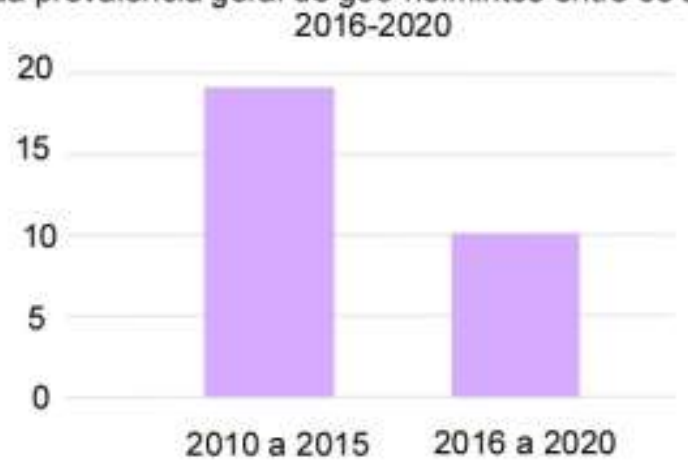

(B) Variaçāo da prevalência combinada por geo-helminto nos últimos 10 anos

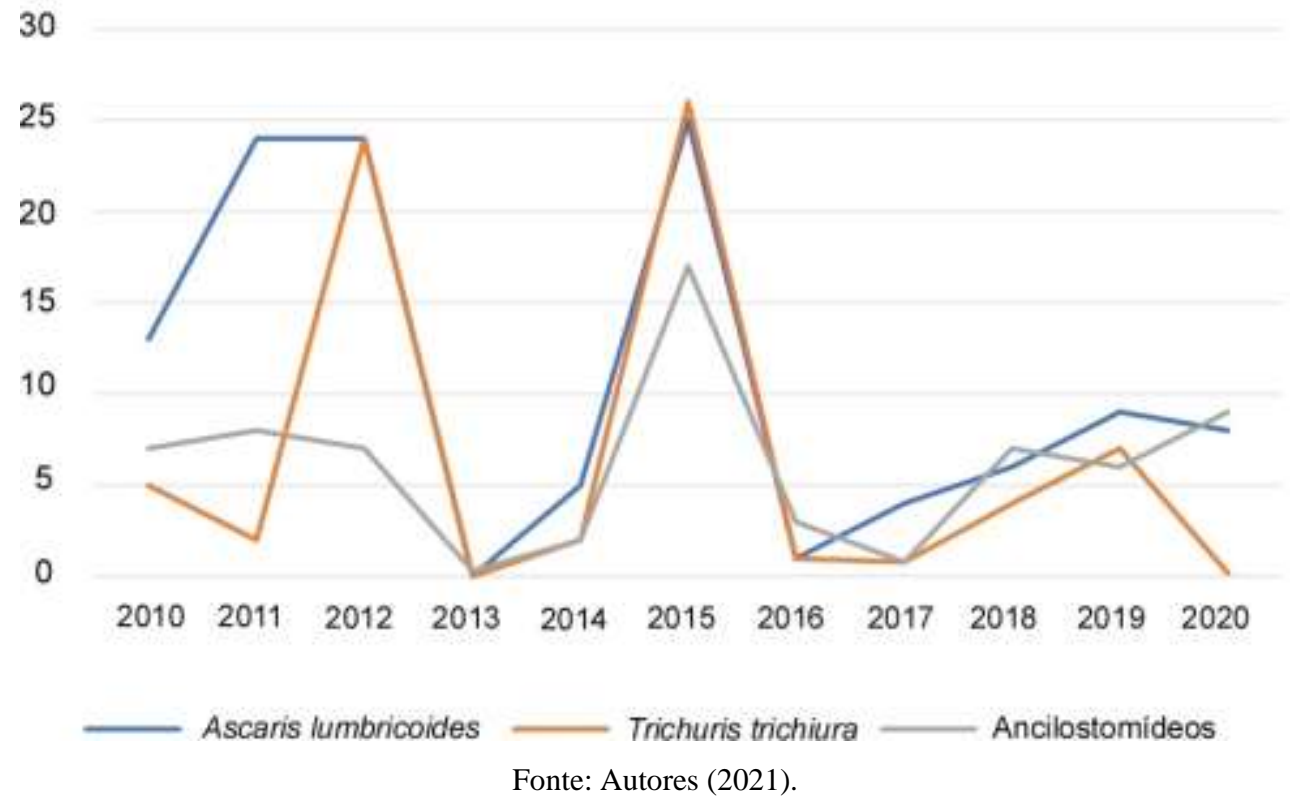

A Figura 4 apresenta dados da variação da prevalência geral combinada de geo-hemintos na população brasileira entre os anos de 2010 a 2020, mostrando uma ligeira queda, e apresenta ainda os dados da variação da prevalência separada por espécie de geo-helminto no mesmo período.

Dos 55 estudos, treze reportaram associações parasitárias de diferentes espécies de geo-helmintos nas amostras humanas (1.231 amostras). A associação dupla entre A. lumbricoides e T. trichiura foi identificada em 15\% (186) das amostras positivas, A. lumbricoides e Ancilostomideos em 2,6\% (32), T. trichiura e Ancilostomídeos em 0,6\% (7) e a associação tripla de A. lumbricoides, T. trichiura, e Ancilostomídeos foi identificada em 9\% (112) das amostras positivas analisadas nos 13 estudos.

Dos estudos analisados, apenas onze distribuíram as amostras segundo o sexo, totalizando 5.653 amostras, das quais 54\% eram do sexo masculino e 46\% masculino. Em total, 1.787 (32\%) estavam positivas. Das amostras positivas, 793 (44\%) eram do sexo feminino e 994 (56\%) masculino.

Vinte e sete estudos distribuíram as amostras por grupos de idade totalizando 35.019 amostras. Destas amostras separadas por idade, 33.631 (96\%) eram crianças de zero a 17 anos e 1.388 (4\%) do grupo de adultos maiores de 18 anos. Destas amostras 12.134 (35\%) resultaram positivas paras infecções por geo-helmintos, sendo 12.039 (99\%) amostras de crianças de zero a 17 anos, 95 (1\%) nos adultos maiores de 18 anos. A análise desses estudos mostra que as crianças de zero a 17 anos são o grupo mais afetado por geo-helmintos no Brasil. 
A idade é um importante fator de risco para infecções parasitárias. De acordo com a Organização Mundial da Saúde (OMS), 568 milhões de crianças em idade escolar vivem em áreas onde geo-helmintos são transmitido de forma intensiva (WHO, 2020). Isso indica que as crianças estão em maior risco de serem infectadas com geo-helmintos. Isso pode estar associado ao fato de as crianças ficarem mais expostas ao solo contaminado quando brincam, andam descalças, comem terra e não têm uma boa higiene pessoal. Os geo-helmintos são responsáveis por infecções crônicas que podem levar ao absenteísmo escolar, retardo do crescimento físico e comprometimento do desenvolvimento cognitivo, particularmente aprendizagem, memória e tempo de reação nas crianças (Pabalan et al., 2018).

\subsection{Fatores associados à infecção por geo-helmintos}

Quarenta e cinco estudos reportaram que os fatores ambientais e socioeconômico podem influenciar diretamente na infecção de geo-helmintos aos humanos. Dentre os trabalhos que avaliaram esse aspecto, $56 \%$ deles reportaram o saneamento precário como um fator correlacionado à infecção por geo-helmintos, $33 \%$ hábitos inadequados de higiene e $24 \%$ baixa renda. $\mathrm{Na}$ Tabela 3 pode-se observar os demais fatores associados às infecções relatadas nos estudos. É importante destacar que muitos desses estudos identificaram mais de um fator associado às infecções por geo-helmintos nas amostras humanas no Brasil.

Tabela 3. Fatores associados às infecções por geo-helmintos apontados nas publicações analisadas.

\begin{tabular}{lcc}
\hline \multicolumn{1}{c}{ Fatores associados } & Publicações - n & \% \\
\hline Saneamento precário & 25 & $56 \%$ \\
Hábitos inadequados de higiene & 15 & $33 \%$ \\
Baixo nível socioeconômico - renda & 11 & $24 \%$ \\
Ausência de programas de educação em saúde & 10 & $22 \%$ \\
Má qualidade da água & 6 & $13 \%$ \\
Baixo nível socioeconômico - escolaridade & 6 & $13 \%$ \\
Ausência de exames de rotina e tratamento & 6 & $13 \%$ \\
Moradia em área rural & 2 & $4 \%$ \\
\hline
\end{tabular}

Fonte: Autores (2021).

$\mathrm{Na}$ Tabela 3 estão destacados todos os fatores associados às infecções por geo-helmintos reportados nos artigos analisados nesta revisão. Os fatores associados estão listados em ordem decrescente das taxas de porcentagens que aparecem nos artigos.

Embora atualmente se use no Brasil o conceito de saneamento como sendo o conjunto de serviços e medidas que incluem abastecimento de água, esgotamento sanitário e manejos de resíduos sólidos para prevenir doenças e melhorar a qualidade de vida da população, o mais comum é que o saneamento seja visto no país como sendo os serviços de acesso à água potável e à coleta e tratamento dos esgotos (Brasil, 2018). A maioria dos artigos analisados nessa revisão apontaram o saneamento precário como um fator correlacionado à infecção por geo-helmintos em diferentes regiões do país, e essa falta de serviços de saneamento cria condições para a transmissão de muitas outras endemias. O saneamento precário foi observado em estudos realizados nas regiões Nordeste (Chaves et al., 2020; Andrade et al., 2018; Rollemberg et al., 2015), Norte (Banhos et al., 2017; Ferraz et al., 2014; Silva et al., 2011; Visser et al., 2011; Oliveira et al., 2010) e Sul (BIOLCHI et al., 2015; MATA- 
Santos et al., 2013; Brandelli et al., 2012), regiões com menores taxas de coleta e tratamento de esgoto, conforme dados do SNIS (Brasil, 2019).

Os hábitos inadequados de higiene foram citados em 33\% dos estudos analisados. Esse aspecto foi observado em diferentes grupos populacionais como moradores de rua em Goiás (Antunes et al., 2020), ribeirinhos do Pantanal (Silva et al.,2018b), comunidade rural na Bahia (Andrade et al., 2018), crianças em Minas Gerais (Moura et al., 2017), população da favela no Rio de Janeiro (Ignácio et al., 2017), catadores de lixo no Distrito Federal (Pereira et al., 2015), indígenas no Mato Grosso (Malheiros et al., 2014), Mato Grosso do Sul (Neres-Norberg et al., 2014) e Rio Grande do Sul (Brandelli et al., 2012), manipuladores de alimentos no Piauí (Fernandes et al., 2014), população de assentamento na Bahia (Santos et al., 2013b), e na população geral em Mato Grosso do Sul (Silva et al., 2019), Bahia (Almeida et al., 2012) e Maranhão e Amapá (Ferraz et al., 2014).

As geo-helmintíases são condições com claros determinantes socioambientais, cuja prevalência é elevada em cenários de pobreza econômica (Weatherhead et al., 2017). Uma significativa taxa dos estudos incluídos nessa revisão (24\%) analisou os dados socioeconômicos da população brasileira, e demonstraram que há uma relação direta entre a infecção por geohelmintos e o nível de renda. Essa alta taxa de associação da renda com as geo-helmintíases pode ser o reflexo de má distribuição de renda no país, que segundo o IBGE (Instituto Brasileiro de Geografia e Estatística), uma grande parcela da população brasileira vive em estado de pobreza.

Uma taxa de 22\% dos estudos incluídos nessa revisão reportou a ausência de programas de educação em saúde como fator associado à infecção por geo-helmintos no Brasil. A transmissão das geo-helmintíases está relacionada aos hábitos, práticas e atitudes da população, portanto, o seu controle requer a participação comunitária com programas integrados com instituições governamentais e sociais, estratégias de saúde da família, escolas e igrejas (Brasil, 2018). Nesse contexto, a Educação em Saúde é fundamental para o desenvolvimento de ações de prevenção e controle das geo-helmintíases.

A importância de condições adequadas de saneamento básico está relacionada ao fornecimento de água potável para a população, uma vez que diversas doenças emergentes e reemergentes no país podem estar associadas à água (BRASIL, 2019). A má qualidade da água foi reportada em 13\% dos estudos como fator associado à infecção por geo-helmintos no Brasil, fato que mostra a necessidade de uma adequada distribuição e tratamento da água para a população.

A educação é a ferramenta mais importante da população na compreensão do processo saúde-doença, capaz de desenvolver nas pessoas a consciência crítica das causas dos seus problemas e, ao mesmo tempo, criar situações para atuar no sentido de mudança. O IBGE apresenta uma taxa de 6,6\% de analfabetismo na população brasileira acima de 15 anos no ano de 2019, e o baixo nível de escolaridade foi reportado como fator associado a infecção por geo-helmintos em 13\% dos estudos incluídos nesta revisão.

Segundo a Organização Mundial de Saúde (WHO, 2012; 2020), o tratamento periódico é uma medida preventiva e efetiva para redução da carga parasitária e disseminação no ambiente. Porém 13\% dos trabalhos analisados reportaram a ausência de exames de rotina e tratamento das geo-helmintíases, na população brasileira, como fator principal na infecção e reinfecção por essas enfermidades. Essa taxa evidencia a necessidade de mais políticas públicas efetivas de tratamento e acesso facilitado aos programas de atenção primária à saúde para toda população. Eustachio et al. (2019) destacaram esse aspecto como um fator importante na infecção por geo-helmintíases em uma população quilombola em Minas Gerais. Também foi reportado em pacientes em hemodiálise de Mato Grosso (Alencar et al., 2020), pacientes com câncer no Rio Grande do Sul (JESKE et al., 2018), indígenas em Minas Gerais (Assis et al., 2013), comunidade ribeirinha no Amazonas (Silva et al., 2012; Valverde et al., 2011), grupos populacionais que encontram dificuldade de acesso aos serviços básicos de saúde. 
Embora menos reportada nos estudos, as áreas rurais também aparecem associadas às infecções por geo-helmintos em $4 \%$ dos estudos, relatadas como desprovidas de serviços de saneamento, favorecendo a infecção nesses locais (Barbosa et al., 2018; Biolchi et al., 2015).

Embora esta revisão tenha gerado dados valiosos sobre a prevalência de geo-helmintos no Brasil, ela tem várias limitações. Primeiro, os dados utilizados não foram derivados de todos os estados brasileiros, portanto, a prevalência combinada de geo-helmintos neste estudo pode não representar totalmente a prevalência no Brasil. Em segundo lugar, as publicações sobre geo-helmintos não foram distribuídas uniformemente entre as cinco regiões. Aproximadamente $25 \%$ dos artigos incluídos foram relatados na região Nordeste, enquanto apenas $14 \%$ na região Centro-Oeste. Terceiro, a maioria dos estudos elegíveis utilizou o exame de fezes simples em vez do exame de fezes múltiplas, conforme recomendado pela OMS, podendo resultar em subestimação da prevalência de geo-helmintos no país. Além disso, apenas 13\% dos estudos utilizaram o método de Kato-Katz recomendado, o que pode levar, também, a subestimar a verdadeira prevalência. Em quarto lugar, muitos estudos analisados nesta revisão não segregaram os resultados das amostras positivas por sexo e/ou idade, bem como muitos não informaram sobre as associações parasitárias, ou ainda, não analisaram os fatores socioambientais relacionados às infecções por geo-helmintos. Isto diminuiu o número das amostras classificadas para avaliar melhor a prevalência por essas variáveis.

\section{Conclusão}

O presente estudo evidenciou um número constante de publicações relacionadas à análise de geo-helmintos na população brasileira nos últimos dez anos, e revelou que as infecções geo-helmínticas ainda são prevalentes no país, embora tenha apresentado uma ligeira queda nos últimos cinco anos. O geo-helminto mais prevalente identificado nas amostras humanas brasileiras seguiu a tendência global, com Ascaris lumbricoides com 68\%, seguido de Ancilostomídeos com 24\% e Trichuris trichiura $8 \%$. Dente os principais fatores associados às infecções por geo-helmintos relatados nas pesquisas, destacam-se o saneamento precário, hábitos inadequados de higiene e a baixa renda. Esperamos que os resultados desta revisão sirvam como uma contribuição importante para os formuladores de políticas públicas e administrações regionais no controle e prevenção de geo-helmintos no país. Este resultado destaca a importância do desempenho das organizações governamentais no processo de sensibilização da população acerca da prevenção e cuidados que devem ser tomados para não propagar a infecção, além de ofertarem um upgrade nos investimentos de infraestrutura tanto no setor de coleta de lixo, rede de esgoto e tratamento de água e expandir a administração em massa de tratamentos antiparasitários no Brasil.

\section{Agradecimentos}

Os autores agradecem o apoio financeiro do Conselho Nacional de Desenvolvimento Científico e Tecnológico - CNPq (Projeto Universal 423391/2018-6).

\section{Referências}

Aguiar-Santos, A. M., Medeiros, Z., Bonfim, C., Rocha, A. C., Brandão, E., Miranda, T., et al. (2013). Epidemiological assessment of neglected diseases in children: lymphatic filiriasis and soil-transmitted helminthiasis. Journal of Pediatrics, 89, 250-5.

Alencar, B. T., Zanetti, A. S., Vilella, S. H., Araújo, M. S. M., Silva, L. N. L. Alencar, R. T., Espinosa, A. O., \& Malheiros, A. F. (2020). Fatores socioambientais e prevalência de enteroparasitas em pacientes em hemodiálise no pantanal mato-grossense, Brasil. Research, Society and Development, 9 , e5109108738.

Almeida, P. H. A., Santana, P. C. S., \& Silva, A. V. (2012). Prevalência de protozoários e helmintos entéricos em residentes de São Cristóvão, Feira de Santana, Bahia, Brasil. Arquivos de Ciências da Saúde UNIPAR, 16, 61-66.

Almeida, M., Monteiro, K., Bacelar, P. et al. (2020). Interactions between malnutrition, soil-transmitted helminthiasis and poverty among children living in periurban communities in Maranhao State, Northeastern Brazil. Journal of the São Paulo Institute of Tropical Medicine, 62(73), 1-9. 
Andrade, R. S., Albuquerque, W. A., Miranda, F. S., Marques, B. C., Mota, L. H. S., Santos, R. S., Silva, I. M. M., \& Amor, A. M. (2018). Presence of enteroparasites in the environment and the resident population in a rural community in Santo Antonio de Jesus in the reconcavo da Bahia, Brazil. Revista de Patologia Tropical, 47, 31-45.

Antunes, R., Souza, A., Xavier, E. et al. (2020). Intestinal parasites: prevalence and epidemiological aspects in homeless people. Original Article RBAC, 52, 87-92.

Assis, E. M., Oliveira, R. C., Moreira, L. E., Pena, J. L., Rodrigues, L. C., \& Machado-Coelho, G. L. L. (2013). Prevalência de parasitos intestinais na comunidade indígena Maxakali, Minas Gerais, Brasil, 2009. Cadernos de Saúde Pública, 29, 681-690.

Banhos, E. F., Rocha, J. A. M., Pimentel, M. L., Batista, E. T. M., \& Silva, L. M. (2017). Prevalence and risk factors for intestinal parasite infections in schoolchildren, in the city of Santarém, Pará state, Brazil. Arquivos Brasileiros de Ciências da Saúde, 42, $137-142$.

Barbosa, V. S., Araújo, K. C., Neto, O. B. L., \& Barbosa, C. S. (2012). Spatial distribution of schistosomiasis and geohelminthiasis cases in the rural areas of Pernambuco, Brazil. Revista da Sociedade Brasileira de Medicina Tropical, 45(5),633-638.

Barbosa, C. V., Barreto, M. M., Andrade, R. J., Sodré, F., d’Avila-Levy, C. M., Peralta, J. M., Igreja, R. P., Macedo, H. W., \& Santos, H. L. C. (2018). Intestinal parasite infections in a rural community of Rio de Janeiro (Brazil): Prevalence and genetic diversity of Blastocystis subtypes. PLoS One, 13, e0193860.

Belloto, M. V. T., Junior, J. E. S., Macedo, E. A., Ponce, A., Galisteu, K. J., Castro, E., Tauyr, L. V., Rossit, A. R. B., \& Machado, R. L. D. (2011). Enteroparasitoses numa população de escolares da rede pública de ensino do município de Mirassol, São Paulo, Brasil. Revista Pan-Amazonica de Saúde, 2, 37-44.

Biolchi, L. C., Collet, M. L., Dallanora, F. J., D’Agostini, F. M., Nardi, G. M., Muller, G. A., \& Wagner, G. (2015). Enteroparaistes and commensals in students aged 7 to 14 years in rural and urban áreas of Campos Novos, west of Santa Catarina, Brazil. Revista de Patologia Tropical, $44,337-342$.

Bragagnoli, G. \& Silva, M. T. N (2014). Ascaris lumbricoides infection and parasite load are associated with asthma in children. Journal Infectious Developing Coutries, 8(7), 891-897.

Branco, N., Leal, D. A. G., \& Franco, R. M. B. (2012). A parasitological survey of natural water springs and inhabitants of a tourist city in southeastern Brazil. Vector-borne and Zoonotic Diseases, 12, 410-417.

Brandelli, C. L. C., De Carli, G. A., Macedo, A. J., \& Tasca, T. (2012). Intestinal parasitism and socio-environmental factors among Mbyá-guarani indians, Porto Alegre, Rio Grande do Sul, Brazil. Revista do Instituto de Medicina Tropical de São Paulo, 54(3),119-122.

Brasil. Ministério da Saúde. (2018). Secretaria de Vigilância em Saúde. Departamento de Vigilância das Doenças Transmissíveis. Guia Prático para o Controle das Geo-helmintíases. Brasília/DF: Ministério da Saúde, 2018, 33 p.

Brasil. Ministério do Desenvolvimento Regional. (2019). Secretaria Nacional de Saneamento Ambiental - SNSA. Sistema Nacional de Informações sobre Saneamento - SNIS: diagnóstico dos serviços de água e esgotos - 2019. Brasília: SNSA/MCIDADES, 2019. http://www.snis.gov.br/diagnostico-anual-agua-eesgotos/diagnostico-dos-servicos-de-agua-e-esgotos-2019.

Brooker, S., Clements, A. C. A., \& Bundy, D. A. P. (2006). Global epidemiology, ecology and control of soil-transmitted helminth infections. Advances in Parasitology, 62, 221-261.

Brooker, S., Hotez, P. J., \& Bundy, D. A. (2008). Hookworm-related anaemia among pregnant women: a systematic review. PLoS neglected tropical diseases, 2(9), e291.

Casavechia, M., Lonardoni, M., \& Venazzi, E. et al. (2016). Prevalence and predictors associated with intestinal infections by protozoa and helminths in southern Brazil. Parasitology Research, 115(6), 2321-2329.

Chammartin, F., Scholte, R. G. C., Guimarães, L. H., Tanner, M., Utzinger, J., \& Vounatsou, P. (2013). Soil-transmitted helminth infection in South America: a systematic review and geostatistical meta-analysis. Lancet Infectious Diseases, 13, 507-518.

Chaves, M., Dantas, S., Silva, A. et al. (2020). Avaliação de variáveis sociodemográficas e da qualidade de vida de portadores de helmintiases intestinais em um hospital universitário. Revista Interdisciplinar de estudos em Saúde da UNIARP, 9, 145-161.

Couto, L. D., Tibiric, S. H. C., Pinheiro, I. O., Mitterofhe, A., Lima, A. C., Castro, M. F., Gonçalves, M., Silva, M. R., Guimarães, R. J. P. S., Rosa, F. M., \& Coimbra, E. S. (2014). Neglected tropical diseases: prevalence and risk factors for schistosomiasis and soil-transmitted helminthiasis in a region of Minas Gerais State, Brazil. Transactions of the Royal Society of Tropical Medicine and Hygiene, 108, 363-371.

Damázio, S. M., Lima, M. S., Soares, A. R., \& Souza, M. A. A. (2013). Intestinal parasites in a quilombola community of the northern state of Espírito Santo, Brazil. Revista do Instituto de Medicina Tropical de São Paulo, 55, 179-183.

Damázio, S. M., Soares, A. R., \& Souza, M. A. A. (2016). Perfil parasitológico de escolares da localidade de Santa Maria, zona rural do município de São Mateus/ES, Brasil. Revista de Atenção Primária a Saúde, 19, 261-267.

Eustachio, P. F. P., Avelar, L. A., Dias, J. V. L., Queiroz, D. R. M., Murta, N. M. G., Oliveira, G. H. B., Cambraia, R. P., Pires, H. H. R., \& Martins, H. R. (2019). Parasitismo intestinal y contaminación ambiental con helmintos y protozoários en una comunidade quilombola del sudeste de Brasil. Revista Cubana de Medicina Tropical, 71, 1-13.

Faria, C. P., Zanini, G. M., Dias, G. S., Silva, S., Freitas, M. B., Almendra, R., Santana, P., \& Sousa, M. C. (2017). Geospatial distribution of intestinal parasitic infections in Rio de Janeiro (Brazil) and its association with social determinats. PLoS Neglected Tropical Diseases, 11 , e0005445. 
Fernandes, N. S., Guimarães, H. R., Amorim, A. C. S., Brito, V. M., Borges, E. P., Reis, M. B., Trindade, R. A., \& Melo, A. C. F. L. (2014). Ocorrência de enteroparasitoses em manipuladores de alimentos de restaurantes em Parnaíba, Piauí-Brasil. Revista de Patologia Tropical, 43, $459-469$.

Ferraz, R. R. N., Barnabé, A. S., Porcy, C., D’Eça Junior, A., Feitosa, T., \& Figueiredo, P. M. (2014). Parasitoses intestinais e baixos índices de Gini em Macapá (AP) e Timon (MA), Brasil. Cadernos de Saúde Coletiva, 22, 173-176.

Ferreira, M. U. (2017). Parasitologia contemporânea. Guanabara Koogan.

Fonseca, E., Teixeira, M., Barreto, M. et al. (2010). Prevalência e fatores associados às geo-helmintíases em crianças residentes em municípios com baixo IDH no Norte e Nordeste brasileiros. Caderno de Saúde Pública, 26(1), 143-152.

Fonseca, R. E. P., \& Barbosa, M. C. R., \& Ferreira, B. R. (2017). High prevalence of enteroparasites in children from Ribeirão Preto, São Paulo, Brazil. Revista Brasileira de Enfermagem, 70, 566-571.

Gyorkos, T. W., \& Gilbert, N. L. (2014). Blood drain: soil-transmitted helminths and anemia in pregnant women. PLoS neglected tropical diseases, 8(7), e2912.

Hailegebriel, T., Nibret, E., \& Munshea, A. (2020). Prevalence of Soil-Transmitted Helminth Infection Among School-Aged Children of Ethiopia: A Systematic Review and Meta-Analysis. Infectious Diseases: Research and Treatment, 13, 1-14.

Iasbik, A. F., Pinto, P. S. A., Guimarães-Peixoto, R. P. M., Santos, T. O., Fernandes, F. M., Silva, L. F., Silva, A. R., Vieira, S. E., \& Araújo, J. V. (2018). Prevalence and transmission of intestinal parasitosis in human beings from zona da mata, Minas Gerais, Brazil. Bioscience Journal, $34,802-809$.

Ignacio, C., Silva, M., Handam, N. e al. (2017). Socioenvironmental conditions and intestinal parasitic infections in Brazilian urban slums: a cross-sectional study. Revista do Instituto de Medicina Tropical de São Paulo, 59, e56.

Jeske, S., Bianchi, T. F., Moura, M. Q., Baccega, B., Pinto, N. B., Berne, M. E. A., \& Villela, M. M. (2018). Intestinal parasites in cancer patients in the South of Brazil. Brazilian Journal of Biology, 78, 574-578.

Karshima, S. N. Prevalence and distribution of soil-transmitted helminth infec- tions in Nigerian children: a systematic review and meta-analysis. Infect Dis Poverty, 7, 69.

Malheiros, A. F., Delgado, P. M., Lemos, L. S., \& Shaw, J. J. (2014). Infection by hookworms in indigenous of a remote region from the legal amazon, brazil. Neotropical Helminthology, 8(2), 403-410.

Mata-Santos, T., Gatti, F. A., Mascarenhas, C. S., Martins, L. H., Mata-Santos, H. A., Fenalti, J. M., Netto, I. C. O., Mendoza-Sassi, R. A., \& Scaini, C. J. (2013). Prevalence of enteroparasites in children atended at basic health unities in a Brazilian southern city. Revista do Instituto Adolfo Lutz, 72 , $175-178$.

Mortean, E. C. M., Falavigna, D. L. M., Janeiro, V., Falavigna-Guilherme, A. L., \& Gomes, M. L. (2012). Low intestinal parasites as an health indicator in a municipality of southern Brazil with intesnive agricultural mechanization. Revista de Saúde e Biologia, 7, 23-29.

Moura, R. G. F., Ramos, E. L. P., Colombo, M. S., Aidar, F. L. M., Hernández, C. G., Silva, M. B. O., \& Oliveira, K. R. (2017). Prevalence of intestinal parasites in child daycare centers: epidemiological significance. Revista de Patologia Tropical, 46, 75-84.

Nagel, A. S., Baccega, B., Hernandes, J. C., Santos, C. V., Gallo, M. C., Quevedo, P. S., \& Villela, M. M. (2017). Intestinal parasite prevalence in shoolchildren from northwestern Rio Grande do Sul state, Brazil. Revista de Patologia Tropical, 46, 277-286.

Neres-Norberg, A., Guerra-Sanches, F., Moreira-Norberg, P. R. B., Madeira-Oliveira, J. T., Santa-Helena, A. A., \& Serra-Freire, N. M. (2014). Enteroparasitismo en indígenas Terena em el Estado de Mato Grosso do Sul, Brasil. Revista de Salud Publica, 16, 859-870.

Neves, D., Melo, A., Linardi, P. et al. (2016). Parasitologia Humana. (13a ed.), Antheneu, 616 p.

Oishi, C. Y., Klisiowicz, D. R., Seguí, R., Köster, P. C., Carmena, D., Toledo, R., Esteban, J. G., Muñoz-Antoli, C. (2019). Reduced prevalence of soiltransmitted helminths and high frequency of protozoan infections in the surrounding urban area of Curitiba, Paraná, Brazil. Parasite Epidemiology and Control, 7, e00115.

Oliveira, C. L. M., Ferreira, W. A., Vasquez, F. G., Barbosa, M. G. V. (2010). Parasitoses intestinais e fatores socioambientais de uma população da área periurbana de Manaus - AM. Revista Brasileira em Promoção da Saúde, 23, 307-315.

Ostan, I., Kilimcioglu, A. A., Girginkardesler, N., Ozyurt, B. C., Limoncu, M. E., Ok, U. Z. (2007). Health inequities: lower socio-economic conditions and higher incidences of intestinal parasites. BMC Public Health. 7(342), 8.

Pabalan, N., Singian, E., Tabangay, L., Jarjanazi, H., Boivin, M. J., Ezeamama, A. E. (2018). Soil-transmitted helminth infection, loss of education and cognitive impairment in school-aged children: a systematic review and meta-analysis. PLoS Neglector Tropical Disease, 12, e0005523.

Pereira, I. G. S., Rodrigues, C. S., Gurgel-Gonçalves, R., Machado, E. R. (2015). Frequency of intestinal parsites and commensals in Street waste pickers from two cooperatives in the Brazilian Federal district. Revista de Patologia Tropical, 44, 432-440.

Periago, M. V., Diniz, R. C., Pinto, S. A., et al. (2015). The right tool for the job: detection of soil-transmitted helminths in areas co-endemic for other helminths. PLoS Neglector Tropical Disease, 9, e0003967.

Reis, L. B., Santos, R. S., Mota, L. H. S., Jesus, J. S. A., Oliveira, J. M. O., Andrade, R. S., Santos, G. A., Amor, A. L. M. (2019). Enteroparasites, demographic profile, socioeconomic status and education level in the rural population of the reconcavo of Bahia, Brazil. Revista de Patologia Tropical, 48(4), 197-210.

Rey, L. et al. (2018). Bases da parasitologia médica. (3a ed.), Guanabara: Anthares. 
Rollemberg, C. V. V., Santos, C. M. B., Silva, M. M. B. L., Souza, A. M. B., Silva, Â. M., Almeida, J.A. P., Almeida, R. P., \& Jesus, A. R. (2011). Aspectos epidemiológicos e distribuição geográfica da esquistossomose e geo-helmintos, no Estado de Sergipe, de acordo com os dados do Programa de Controle da Esquistossomose. Revista da Sociedade Brasileira de Medicina Tropical, 44(1), 91-96.

Rollemberg, C. V. V., Silva, M. M. B. L., Rollemberg, K. C., Amorim, F. R., Lessa, N. M. N., Santos, M. D. S., Souza, A. M. B., Melo, E. V., Almeida, R. P., Silva, A. M., Werneck, G. L., Santos, M. A., Almeida, J. A. P., \& Jesus, A. R. (2015). Predicting frequency distribution and influence of sociodemographic and behavioral risk factors of Schistosoma mansoni infection and analysis of co-infection with intestinal parasites. Geospatial Health, 10, 13-19.

Santos, F. L. N., Souza, A. M. G. C., \& Soares, N. M. (2013a). Hookworm and threadworm infections and their association with hemoglobin and eosinophil concentrations in residents of Salvador-Bahia, Brazil. Revista do Instituto de Medicina Tropical de São Paulo, 55(4), 233-238.

Santos, H. L. C., Martins, L. A. F., Peralta, R. H. S., Peralta, J. M., \& Macedo, H. W. (2013b). Frequency of amoebiasis and other intestinal parasitosis in a settlement in Ilhéus city, state of Bahia, Brazil. Revista da Sociedade Brasileira de Medicina Tropical, 47, 11-14.

Seguí, R., Muñoz-Antoli C., Klisiowicz, D. R., Oishi, C. Y., Köster, P. C., Lucio, A., Hernández-de-Mingo, M., Puente, P., Toledo, R., Esteban, J. G., \& Carmena, D. (2018). Prevalence of intestinal parasites, with emphasis on the molecular epidemiology of Giardia duodenalis and Blastocystis sp., in the Paranaguá Bay, Brazil: a community survey. Parasites \& Vectors, 11, 490.

Seixas, M. T. L., Souza, J. N., Souza, R. P., Teixeira, M. C. A., \& Soares, N. M. (2011). Avaliação da frequência de parasitos intestinais e do estado nutricional em escolares de uma área periurbana de salvador, Bahia, Brasil. Revista de Patologia Tropical, 40, 304-314.

Silva, L. P. \& Silva, R. M. G. (2010). Ocorrência de enteroparasitos em centros de educação infantil no município de Patos de Minas, MG, Brasil. Biocience Journal, 26, 147-151.

Silva, F. S., Paulo, A. D. C., Braga, C. M. M., Almeida, R. J., \& Galvão, V. P. (2010). Frequência de parasitos intestinais no município de Chapadinha, Maranhão, Brasil. Revista de Patologia Tropical, 39, 63-68.

Silva, J. C., Furtado, L. F. V., Ferro, T. C., B., Carvalho, K., Borges, E. P., \& Melo, A. C. F. L. (2011). Parasitismo por Ascaris lumbricoides e seus aspectos epidemiológicos em crianças do Estado do Maranhão. Revista da Sociedade Brasileira de Medicina Tropical, 44(1), 100-102.

Silva, E. F., Silva, V. B. C., \& Freitas, F. L. C. (2012). Parasitoses intestinais em crianças residentes na comunidade ribeirinha São Francisco do Laranjal, município de Coari, estado do Amazonas, Brasil. Revista de Patologia Tropical, 41, 97-101.

Silva, J. S. H., Silva, D. J., Shaw, J. J., \& Malheiros, A. F. (2018a). Prevalência de enteroparasitos em moradores da cidade de Cáceres/MT. Revista Ibero Americana de Ciências Ambientais, 9, 154-164.

Silva, P., Maciel, L., Castro, L. et al. (2018b). Enteroparasites in Riverside Settlements in the Pantanal Wetlands Ecosystem. Journal of Parasitology Research, e6839745, 1-5.

Silva, R. S. B., Malheiros, A. F., Santos, D. P., Shaw, J. J., Araújo, M. S. M., Moraes, M. F. A., \& Campos, W. N. L. (2019). Estudo de parasitoses intestinais em moradores de Corumbá, Mato Grosso do Sul, Brasil. Revista Ibero Americana de Ciências Ambientais, 10, 109- 128.

Silva, J., \& Rocha, T. (2019). Frequency of helminths according to the data of the program of control of schistosomiasis in the municipality of Xexéu, Pernambuco. Journal Health Biological Sciences, 7(3), 253-257.

Silver, Z. A., Kaliappan, S. P., Samuel, P., et al. (2018). Geographical distribution of soil- transmitted helminths and the effects of community type in South Asia and South East Asia - a systematic review. PLoS Neglector Tropical Disease, 12, e0006153.

Soares, C. B. et al. (2014). Revisão integrativa: conceitos e métodos utilizados na enfermagem. Revista da escola de enfermagem da USP, 48(2), 335-345.

Stoltzfus, R. J., Kvalsvig, J. D., Chwaya, H. M., Montresor, A., Albonico, M., Tielsch, J. M., et al. (2001). Effects of iron supplementation and anthelmintic treatment on motor and language development of preschool children in Zanzibar: double blind, placebo controlled study. British Medical Journal (Clinical research edition), 323(7326), 1389-93.

Valverde, J. G., Gomes-Slva, A., Moreira, C. J. C., Souza, D. L., Jaeger, L. H., Martins, P. P., Meneses, V. F., Boia, M. N., \& Carvalho-Costa, F. A. (2011). Prevalence and epidemiology of intestinal parasitism, as revealed by three distinct techniques in an endemic area in the Brazilian Amazon. Annals of Tropical Medicine e Parasitology, 105, 413-424.

Visser, S., Giatti, L. L., Carvalho, R. A. C., \& Guerreiro, J. C. H. (2011). Estudo da associação entre fatores socioambientais e prevalência de parasitose intestinal em área periférica da cidade de Manaus (AM, Brasil). Ciência \& Saúde Coletiva, 16(8), 3481-3492.

Weatherhead, J., Hotez, P., \& Mejia, R. (2017). The global state of helminth control and elimination in children. Pediatric Clinics of North America Journal, $64,867-77$

Whittemore, R. \& Knafl, K. (2005). The integrative review: updated methodology. Journal of Advanced Nursing, 52(5), 546-53.

WHO - Word Health Organzation. (1994). Bench aids for the diagnosis of intestinal parasites. Geneva, Switzerland: World Health Organzation.

WHO - Word Health Organzation. (2012). Soil-transmitted helminthiases: eliminating soiltransmitted helminthiases as a public health problem in children: progress report 2001-2010 and strategic plan 2011-2020. Geneva, Switzerland: World Health Organzation.

WHO - Word Health Organzation. (2013). Assessing the Efficacy of Anthelminthic Drugs against Schistosomiasis and Soil-Transmitted Helminthiases. Geneva, Switzerland: World Health Organzation.

WHO - Word Health Organzation. (2020). Soil-transmitted helminth infections. https://www.who.int/newsroom/fact-sheets/detail/soil-transmitted-helminthinfections. 